\title{
REPRESENTATION OF MIRRORS IN BEAM WAVEGUIDES AS INCLINED PHASE-TRANSFORMING SURFACES
}

\author{
S. WITHINGTON,' J. A. MURPHY' and K. G. ISAAK \\ 'Cavendish Laboratory, University of Cambridge, Cambridge, U.K. and \\ 'St Patrick's College, Maynooth, Ireland
}

(Received 13 April 1994)

\begin{abstract}
We have developed an approximate technique, based on the principles of multi-mode Gaussian optics. for studying the behaviour of shaped off-axis mirrors. We describe a mirror as an inclined phase-transforming plane, where the phase transformation across the plane is determined by the depth of the mirror as a function of position. The scattering matrix is calculated in the usual way by evaluating the overlap integrals over some surface for which the amplitudes and phases of the incoming and outgoing fields are known; because, however, the modes are not orthogonal over the surface of interest, a system of linear equations has to be solved. We demonstrate the "thin-mirror" technique by studying the behaviour of paraboloidal and ellipsoidal mirrors, and we show how the performance of measured and approximate surfaces can be assessed.
\end{abstract}

\section{INTRODUCTION}

Systems of shaped mirrors are used extensively at submillimetre wavelengths for controlling the characteristics of free-space beams. To analyse such systems, one can either throw away detailed information regarding the image-forming properties of the beam and propagate the scale size in the form of a weakly-diffracting Gaussian mode, ${ }^{(1.2)}$ or one can use classical reflector-antenna theory to calculate the detailed forms of the reflected fields in terms of the geometrical projections of the incident fields. ${ }^{(3.4)}$ The first method is an approximate way of analysing the behaviour of systems of mirrors; whereas, the second method is a precise way of calculating the performance of individual mirrors. The second method can, of course, be applied to systems, but the emphasis on numerical rigour tends to obscure the basic physical processes involved. In this paper, we presented an approximate technique, based on the principles of multimode Gaussian optics, for studying the behaviour of shaped off-axis mirrors. The complexity of the technique is intermediate between the above two extremes, and therefore, the scheme provides a useful insight into the way systems of mirrors work.

By analogy with a thin lens, we represent an offset reflector as an inclined phase-transforming plane. The phase transformation across the plane, which is determined by the depth of the mirror as a function of position, can be any arbitrary function allowing paraboloidal, ellipsoidal, or other forms to be analysed. The mirror is characterized by the way in which it scatters power between high-order Gaussian modes.

Because the surface of the mirror is described in a simple way, the technique can be put to a variety of uses. For example, when ellipsoidal mirrors are used at frequencies away from the nominal design frequency, phase errors occur which can limit the efficiency of a system; ${ }^{(5.6)}$ our technique can be used to model this effect. In addition, the technique can be used to assess the efficiency of measured or approximate surfaces. In array receivers most of the beams pass through the optics off axis, and one has to worry about the distortions of individual beams in addition to the large-scale aberrations of the image. At the present time, we are particularly interested in applying phase-retrieval methods to submillimetre-wave optics, ${ }^{(7)}$ and in this case one has to be certain that the mirror used to defocus the beam does not scatter power between Gaussian modes. 


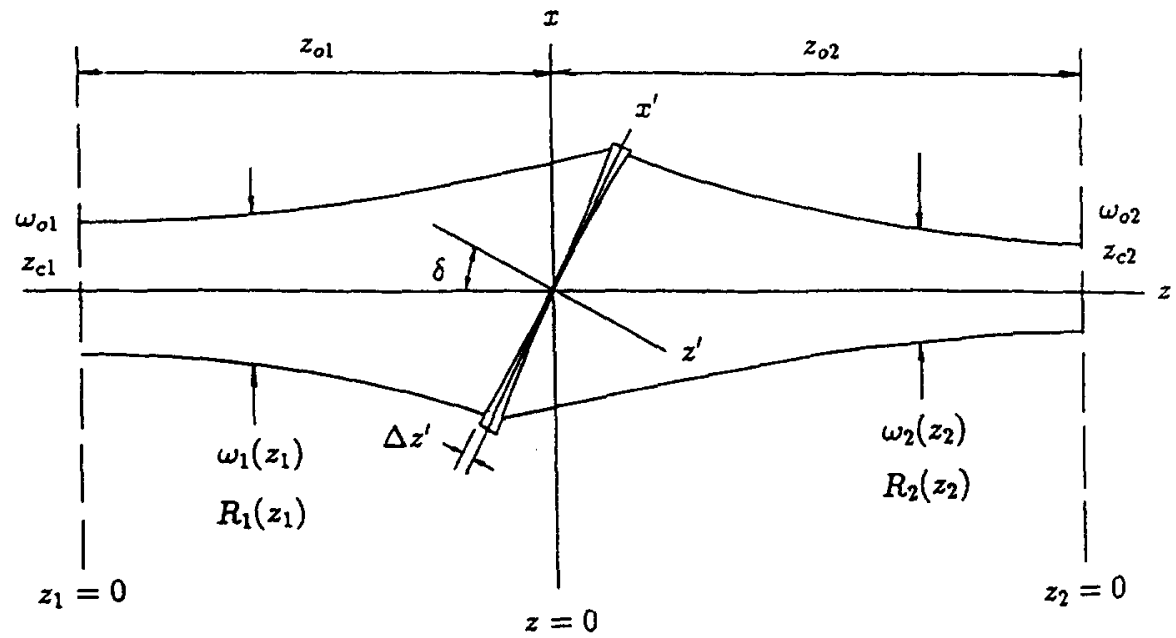

Fig. 1. The reflection of a Gaussian beam by an offset reflector. The beam is shown as though in transmission.

\section{THIN MIRRORS}

The co-ordinate system to be used in the analysis is shown in Fig. 1, and a detailed view of the mirror is shown in Fig. 2. The mirror is shown as though in transmission, and the plane tangent to the surface at the position of the optical axis is used to separate the incoming and outgoing Gaussian beams. If a generic point in the $(x, y, z)$ frame is denoted by $s$, and a point in the, inclined, $\left(x^{\prime}, y^{\prime}, z^{\prime}\right)$ frame is denoted by $s^{\prime}$, then the two frames are connected on the input surface by

$$
\begin{aligned}
& x=x^{\prime} \cos \delta+\Delta z^{\prime} \sin \delta=x_{0}+\Delta x \\
& y=y^{\prime}=y_{o} \\
& z=x^{\prime} \sin \delta-\Delta z^{\prime} \cos \delta=z_{o}-\Delta z
\end{aligned}
$$

and on the output surface by

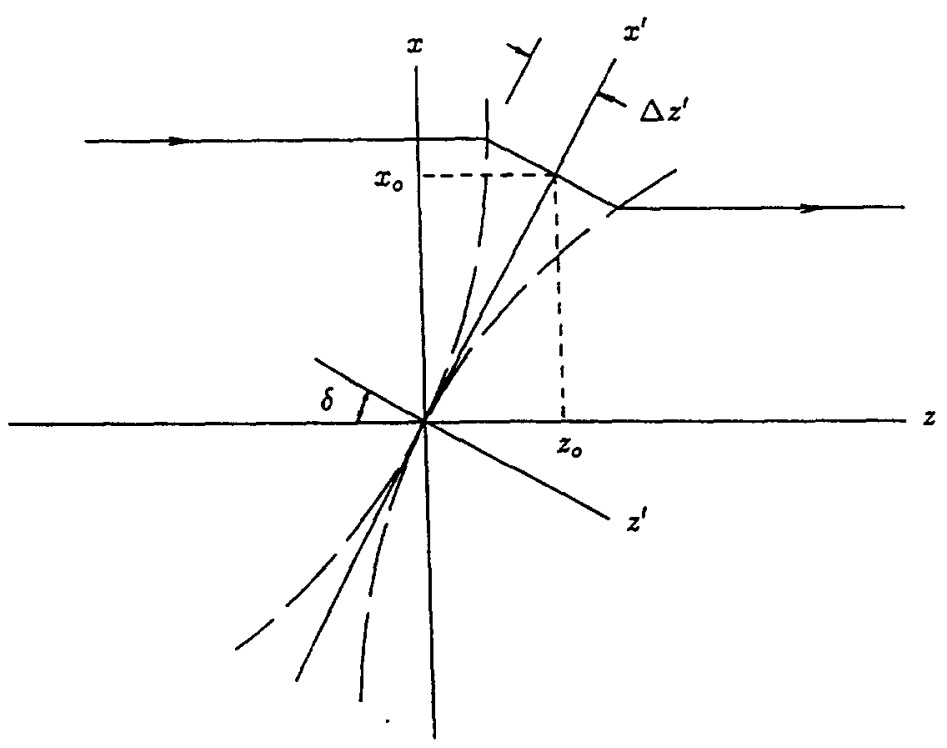

Fig. 2. A mirror is shown as an inclined phase-transforming plane, where the phase transformation across the plane is determined by the depth of the mirror $\Delta z^{\prime}$ as a function of position $\left(x^{\prime}, y^{\prime}\right)$. 


$$
\begin{aligned}
& x=x^{\prime} \cos \delta-\Delta z^{\prime} \sin \delta=x_{0}-\Delta x \\
& y=y^{\prime}=y_{0} \\
& z=x^{\prime} \sin \delta+\Delta z^{\prime} \cos \delta=z_{0}+\Delta z,
\end{aligned}
$$

where the form of the mirror is described by the height of the surface above the tangent plane: $\Delta z^{\prime}=f\left(x^{\prime}, y^{\prime}\right)$. Extracting the plane-wave dependence, the fields incident on and reflected from point $s^{\prime}$ can be written as

$$
E_{i}\left(s^{\prime}\right) \exp \left[-j k\left(z_{o}-\Delta z^{\prime} \cos \delta\right)\right] \text { and } E_{o}\left(s^{\prime}\right) \exp \left[-j k\left(z_{o}+\Delta z^{\prime} \cos \delta\right)\right],
$$

respectively. Because these points represent the same physical position, we have

$$
E_{o}\left(s^{\prime}\right)=E_{i}\left(s^{\prime}\right) \exp \left[j k 2 \Delta z^{\prime} \cos \delta\right]=E_{i}\left(s^{\prime}\right) \exp \left[j \Delta \phi\left(x^{\prime}, y^{\prime}\right)\right] .
$$

The mirror is thus being described as an inclined phase-transforming surface, and the obliquity factor enters naturally as expected.

Because our technique is not intended as a way of calculating precisely the losses that occur in multiple-mirror systems, we ignore cross-polar scattering and represent the fields as scalar functions. For long-focal-length optics, the input and output beams can be decomposed into sums of propagating free-space modes:

$$
E_{i}\left(s^{\prime}\right)=\sum_{m . n} A_{m n} \psi_{m n}^{i}\left(s^{\prime}\right) \quad \text { and } \quad E_{o}\left(s^{\prime}\right)=\sum_{r . s} B_{r s} \psi_{r s}^{o}\left(s^{\prime}\right)
$$

To determine the mode coefficients, we evaluate the overlap integrals over the surface of the mirror $M$ in the inclined output co-ordinate frame. Unlike usual analyses, however, the modes are not orthonormal over the surface of interest; nevertheless

$$
\iint_{M} E_{o}\left(s^{\prime}\right) \psi_{i j}^{o^{*}}\left(s^{\prime}\right) \mathrm{d} M=\iint_{T} E_{o}\left(s^{\prime}\right) \psi_{i j}^{o^{*}}\left(s^{\prime}\right)\left[1+\left(\frac{\partial f}{\partial x^{\prime}}\right)^{2}+\left(\frac{\partial f}{\partial y^{\prime}}\right)^{2}\right]^{1 / 2} \mathrm{~d} x^{\prime} \mathrm{d} y^{\prime},
$$

where $T$ is the orthogonal projection of $M$ onto the tangent plane. For thin mirrors, the corrections to the elemental areas are small,

$$
\left(\frac{\partial f}{\partial x^{\prime}}\right)^{2} \ll 1 \text { and }\left(\frac{\partial f}{\partial y^{\prime}}\right)^{2} \ll 1 \text {, }
$$

and the integral is easy to evaluate numerically. For convenience, we project the integral onto the $z=0$ plane, by making the substitution $x^{\prime}=x_{0} / \cos \delta$ and $y^{\prime}=y_{o}$, to give the $s_{o}$ frame $\left(x_{o}, y_{o}, z_{o}\right)$. For most mirrors this new surface is circular, but for our purposes the integral will be taken to infinity. Finally, the overlap integral becomes

$$
\int_{-\infty}^{+\infty} \int_{-x}^{+\infty} E_{o}\left(s_{o}\right) \psi_{i j}^{o^{*}}\left(s_{o}\right) J \mathrm{~d} x_{o} \mathrm{~d} y_{o},
$$

where the Jacobian $J$ is defined in the usual way, and because the mirror slopes in the $x$ direction, $J=\sec \delta=\sqrt{1+\tan ^{2} \delta}$ : as shown on Fig. $1, \delta$ is the angle of incidence.

We can substitute the modal expansion into the overlap integrals to get

$$
\iint_{M} E_{o}\left(s^{\prime}\right) \psi_{i j}^{0^{*}}\left(s^{\prime}\right) \mathrm{d} M=\sum_{p s} B_{r s} \beta_{i j r} \sec \delta
$$


where

$$
\beta_{i j s}=\int_{-\infty}^{+\infty} \int_{-\infty}^{+\infty} \psi_{r s}^{o}\left(s_{o}\right) \psi_{i j}^{0^{*}}\left(s_{o}\right) \mathrm{d} x_{o} \mathrm{~d} y_{o} .
$$

Through equation (4), we can express the reflected field in terms of the incident field and write

$$
\iint_{M} E_{o}\left(s^{\prime}\right) \psi_{i j}^{o^{*}}\left(s^{\prime}\right) \mathrm{d} M=\int_{-\infty}^{+\infty} \int_{-\infty}^{+\infty} E_{i}\left(s_{o}\right) \exp \left[j \phi\left(x_{o} / \cos \delta, y_{o}\right)\right] \psi_{i j}^{o^{*}}\left(s_{o}\right) J \mathrm{~d} x_{o} \mathrm{~d} y_{o} .
$$

Again, substituting the modal expansion leads to

$$
\iint_{M} E_{o}\left(s^{\prime}\right) \psi_{i j}^{o^{*}}\left(s^{\prime}\right) \mathrm{d} M=\sum_{m, n} A_{m n} \alpha_{i j m n} \sec \delta
$$

where

$$
\alpha_{i j m n}=\int_{-\infty}^{+\infty} \int_{-\infty}^{+\infty} \psi_{m n}^{i}\left(s_{o}\right) \exp \left[j \Delta \phi\left(x_{o} / \cos \delta, y_{o}\right)\right] \psi_{i j}^{*^{*}}\left(s_{o}\right) \mathrm{d} x_{o} \mathrm{~d} y_{o} .
$$

Finally, combining these two results we find, for all $i j$,

$$
\sum_{r, s} B_{r s} \beta_{i j r s}=\sum_{m, n} A_{m n} \alpha_{i j m n}
$$

Clearly, this set of equations can be expressed in matrix form: $\alpha \mathbf{A}=\boldsymbol{\beta} \mathbf{B}$, where $\mathbf{A}$ and $\mathbf{B}$ are vectors of mode coefficients, $\alpha$ is a matrix describing the scattering of power between modes, and $\beta$ is a matrix describing the non-orthogonality of the modes across surface of the mirror. By solving this system of linear equations, we can calculate a scattering matrix $\mathbf{S}$, which relates the mode coefficients of the input beam to the mode coefficients of the output beam:

$$
\mathbf{B}=\boldsymbol{\beta}^{-1} \boldsymbol{\alpha} \mathbf{A}=\mathbf{S A} \text {. }
$$

Let us choose propagating Hermite polynomials as the basis set. A suitable expansion function is

$$
\psi_{i j}=\frac{\sqrt{2}}{w(z)} h_{i}\left(\frac{\sqrt{2} x}{w(z)}\right) h_{j}\left(\frac{\sqrt{2} y}{w(z)}\right) \exp \left[-j \frac{\pi}{\lambda R(z)}\left(x^{2}+y^{2}\right)\right] \exp \left[j(i+j+1) \tan ^{-1} \frac{z}{z_{c}}\right] \exp [-j k z],
$$

where

$$
h_{m}(u)=\frac{H_{m}(u) \exp \left[-\frac{u^{2}}{2}\right]}{\left(\sqrt{\pi} 2^{m} m !\right)^{1 / 2}} .
$$

This set is normalized in the sense that the total power is unity. The symbols have their usual meaning:(1) $w(z)$ is the Gaussian radius at $z, R(z)$ is the mode phase-front radius of curvature at $z$, and $z_{c}$ is the confocal distance. As shown in Fig. 1, the input beam is described in terms of coordinate $z_{1}$, and the output beam is described in terms of coordinate $z_{2}$. Moreover, the input modes have their waists at $z_{1}=0$, and the output modes have their waists at $z_{2}=0$; consequently, the relationship between the two coordinate frames is $z_{1}=z_{2}+z_{a 1}+z_{02}$. Clearly, the tangent plane is described in the input frame by $z_{1}=x_{0} \tan \delta+z_{o 1}$ and in the output frame by $z_{2}=x_{o} \tan \delta-z_{o 2}$. 
The parameters of the output beam are chosen so that the waists at the centre of the mirror are equal:

$$
w_{1}\left(z_{o 1}\right)=w_{2}\left(z_{o 2}\right),
$$

and after taking into account the focusing effect of the mirror, the radii of curvature match:

$$
\frac{1}{R_{1}\left(z_{o 1}\right)}+\frac{1}{R_{2}\left(z_{o 2}\right)}=\frac{1}{f}
$$

where $f$ is the focal length. In some cases, it may be difficult to decide exactly what the value of $f$ should be. For example, an off-axis spherical mirror is astigmatic, and the sagittal and tangential focal lengths are different.

The position and size of the output waist can be found by satisfying the above two requirements, ${ }^{(1)}$ and this leads to

$$
\frac{z_{o 2}}{f}=1+\frac{\left(z_{o 1} / f\right)-1}{\left[\left(z_{o 1} / f\right)-1\right]^{2}+\left(\pi w_{o l}^{2} / \lambda f\right)^{2}}
$$

and

$$
\left(\frac{w_{o 2}}{w_{o 1}}\right)^{2}=\frac{1}{\left[\left(z_{o 1} / f\right)-1\right]^{2}+\left(\pi w_{o 1}^{2} / \lambda f\right)^{2}}
$$

where $w_{o 1}$ and $w_{o 2}$ are the waists of the input and output modes, respectively. For later use. we will define $z_{c 1}$ and $z_{c 2}$ to be the confocal distances of the input and output modes.

Substituting the mode set into equation (10) gives an integral, which can be evaluated as it stands, or various levels of approximation can be introduced. For brevity, we will assume that all of the offsets, $\Delta x$ and $\Delta z$, can be ignored when calculating the $\beta$ matrix. This is equivalent to following the tangent plane, rather than the surface of the mirror, when calculating the amplitude and the dispersion phase. Clearly, this approximation is reasonable if the mirror is shallow. Making this approximation, we find

$$
\beta_{i j r s}=\int_{-\infty}^{+\infty} \frac{\sqrt{2}}{w_{2}\left(z_{2}\right)} h_{r}\left(\frac{\sqrt{2} x_{0}}{w_{2}\left(z_{2}\right)}\right) h_{i}\left(\frac{\sqrt{2} x_{o}}{w_{2}\left(z_{2}\right)}\right) \exp \left[j(r-i) \tan ^{-1} \frac{z_{2}}{z_{c 2}}\right] \mathrm{d} x_{o} \delta_{j s},
$$

where we have taken advantage of the fact that, because the mirror is inclined in the $x$ direction, the modes in the $y$ direction remain orthogonal. As a consequency, $\beta$ becomes a block diagonal matrix; a feature which can be exploited when the matrix is inverted $-\delta_{j s}$ is the Dirac $\delta$-function.

It becomes apparent, when performing analyses, that in many cases the modes are almost orthogonal even when $\delta=45^{\circ}$; when coupling does occur, power is coupled into modes having similar orders, and this implies that some form of perturbation analysis would be appropriate. ${ }^{(8)}$ It also means that by ignoring second-order terms, the $\boldsymbol{\beta}$ matrix can be inverted without recourse to full substitution methods.

We can also substitute the mode set into equation (13) to give an equation, which cannot be simplified in the general case. It would be straightforward to evaluate numerically, but in this paper, we again follow the tangent plane when calculating the field amplitude and the dispersion phase: it is of course important to follow the surface of the mirror when evaluating the curvature term. This approximation leads to

$$
\begin{aligned}
\alpha_{i j m n}= & \int_{-\infty}^{+x} \frac{2}{w_{1}\left(z_{1}\right) w_{2}\left(z_{2}\right)} h_{m}\left(\frac{\sqrt{2}\left(x_{o}\right)}{w_{1}\left(z_{1}\right)}\right) h_{i}\left(\frac{\sqrt{2}\left(x_{o}\right)}{w_{2}\left(z_{2}\right)}\right) \exp \left[j(m+n+1) \tan ^{-1} \frac{z_{1}}{z_{c 1}}\right] \\
& \times \exp \left[-j(i+j+1) \tan ^{-1} \frac{z_{2}}{z_{c 2}}\right] \int_{-\infty}^{+x} h_{n}\left(\frac{\sqrt{2} y_{o}}{w_{1}\left(z_{1}\right)}\right) h_{j}\left(\frac{\sqrt{2} y_{o}}{w_{2}\left(z_{2}\right)}\right)
\end{aligned}
$$




$$
\begin{aligned}
& \times \exp \left[-j \frac{\pi}{\lambda R_{1}\left(z_{1}-\Delta z\right)}\left(\left(x_{o}+\Delta x\right)^{2}+y_{o}^{2}\right)\right] \\
& \times \exp \left[+j \frac{\pi}{\lambda R_{2}\left(z_{2}+\Delta z\right)}\left(\left(x_{o}-\Delta x\right)^{2}+y_{o}^{2}\right)\right] \exp \left[j \Delta \phi\left(x^{\prime}, y^{\prime}\right)\right] \mathrm{d} y_{o} \mathrm{~d} x_{o} .
\end{aligned}
$$

To complete the analysis we need to modify the last equation. A problem occurs because the last three terms, which constitute the primary phase error across the surface of the mirror, are based on the paraxial approximation, and as a consequence, the long-wavelength behaviour is described correctly, but the short-wavelength behaviour is not. We can correct this deficiency, albeit in a heuristic manner, by replacing the parabolic phase front of the Gaussian mode with a spherical phase front-we do not use the more complicated wide-angle modes suggested by Touvinen. ${ }^{(9)}$ The approximate phase factor is given by

$$
\begin{aligned}
& \exp \left[-j\left(\frac{2 \pi}{\lambda}\right) \frac{\left(x_{0}+\Delta x\right)^{2}+y_{o}^{2}}{R_{1}\left(z_{1}-\Delta z\right)+\sqrt{R_{1}^{2}\left(z_{1}-\Delta z\right)+\left(x_{0}+\Delta x\right)^{2}+y_{0}^{2}}}\right] \\
& \exp \left[+j\left(\frac{2 \pi}{\lambda}\right) \frac{\left(x_{o}-\Delta x\right)^{2}+y_{o}^{2}}{R_{2}\left(z_{2}+\Delta z\right)-\sqrt{R_{2}^{2}\left(z_{2}+\Delta z\right)+\left(x_{0}-\Delta x\right)^{2}+y_{0}^{2}}}\right]
\end{aligned}
$$

Once $\alpha$ and $\beta$ are known, the scattering matrix can be calculated through equations (15).

\section{PARABOLIC MIRRORS}

Parabolic mirrors are often used in quasioptical systems, although strictly speaking, they should only be used at very short wavelengths. That is to say, a parabolic mirror converts a beam having a given phase-front radius of curvature into a plane wave. If the focal length of the mirror is set equal to the actual radius of curvature of the incoming phase front, the output waist will occur at the mirror and the outgoing beam will diffract away from the mirror. If, however, the focal length is set equal to the distance from the input waist, the output waist will occur at a focal length beyond the mirror, and phase errors will occur as a consequence of the paraboloid having only one characteristic radius of curvature. We can investigate the associated reduction in efficiency by using the "thin-mirror" technique.

To perform an analysis, we must find the height of the surface above the tangent plane. The geometry is shown in Fig. 3. If the focal length of the mirror is $f$ then the focal length of the parent paraboloid is $a=f \cos ^{2} \delta$. We also require the coordinates of the centre of the mirror: $X_{o}=f \sin (2 \delta)$ and $Z_{o}=f \cos (2 \delta)$. By shifting and rotating the $(X, Z)$ co-ordinate frame, it is possible to show that the thickness of the mirror is given by

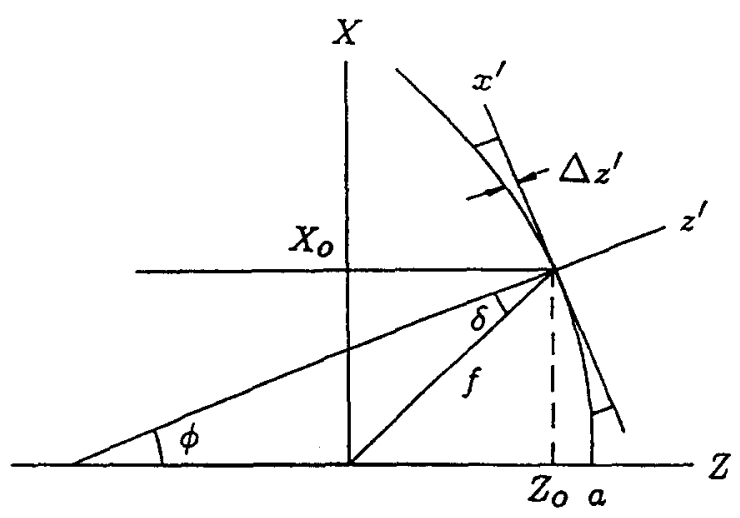

Fig. 3. The geometry of an offset paraboloidal reflector. 


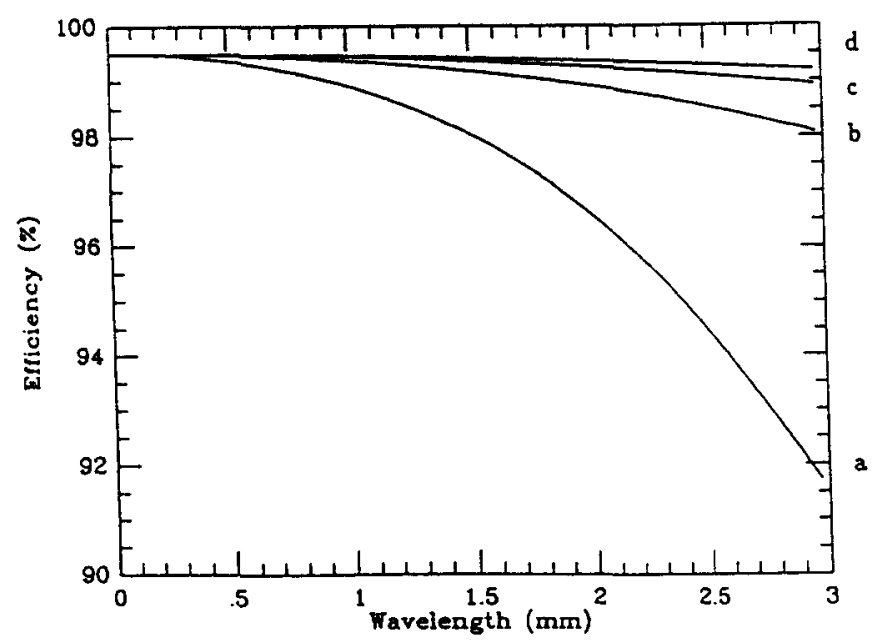

Fig. 4. The coupling efficiency of the lowest-order Gaussian mode as a function of wavelength. The plots correspond to offset paraboloidal reflectors having focal lengths of (a) $50 \mathrm{~mm}$, (b) $100 \mathrm{~mm}$, (c) $150 \mathrm{~mm}$ and (d) $200 \mathrm{~mm}$. The focal ratio of the illuminating beam is held constant at five, the input waist is at the focus, and angle of incidence is $45^{\text {: }}$.

$$
\Delta z^{\prime}=\left|\frac{-B+\sqrt{B^{2}-4 A C}}{2 A}\right| .
$$

where

$$
\begin{aligned}
& A=\sin ^{2} \delta \\
& B=2 x^{\prime} \sin \delta \cos \delta+2 \sin \delta X_{o}+4 a \cos \delta \\
& C=x^{12} \cos ^{2} \delta+2 x^{\prime} \cos \delta X_{o}-4 a x^{\prime} \sin \delta+y^{\prime 2} .
\end{aligned}
$$

We have used the above scheme to calculate the behaviour of a number of offset parabolic reflectors: in all cases, the angle of incidence was chosen to be $45^{\circ}$. In Fig. 4 , we show the coupling efficiency of the lowest-order Gaussian mode as a function of wavelength for mirrors having focal lengths of 50,100,150 and $200 \mathrm{~mm}$. In each case, the input waist was placed at the focus of the mirror, and the focal ratio of the input beam was held constant at $f b=5$. That is to say, we scaled the waist with frequency according to $w_{o 1}=2 \lambda f b / \pi$. This situation occurs, for example, when a horn is used to illuminate a reflecting antenna, in a frequency-independent way, through a Gaussianbeam telescope.

As expected, the beams are well matched at short wavelengths, but at long wavelengths phase errors occur, which reduce the efficiency of the system. By calculating the loss with the principal phase-error term in equation (23) set to zero, the losses due to projection effects can, to some extent, be separated from those due to phase mismatch. In reality, the projection losses are less than those calculated in the above way, because we chose to follow the tangent plane rather than the surface of the mirror when evaluating the overlap integrals. If one follows, the surface of the mirror, then the projection losses do indeed decrease, by some small amount, as expected.

When calculating the performance of mirrors as a function of wavelength in the above way, one must be careful to ensure that the focal ratios of the input and output beams do not become too small. If the focal ratio of one of the beams does become small, the errors incurred by following the tangent plane rather than the surface of the mirror can become insignificant. In our work, we have not been concerned about over estimating the loss in these extreme cases, because we have taken the view that one would not be using such mirrors anyway. To recognize these situations, it should be appreciated that the input and output focal ratios are related by $f b_{1} f b_{2}=\pi f / 4 \lambda$. Problems are more likely to arise at long wavelengths when the product of the focal ratios is 


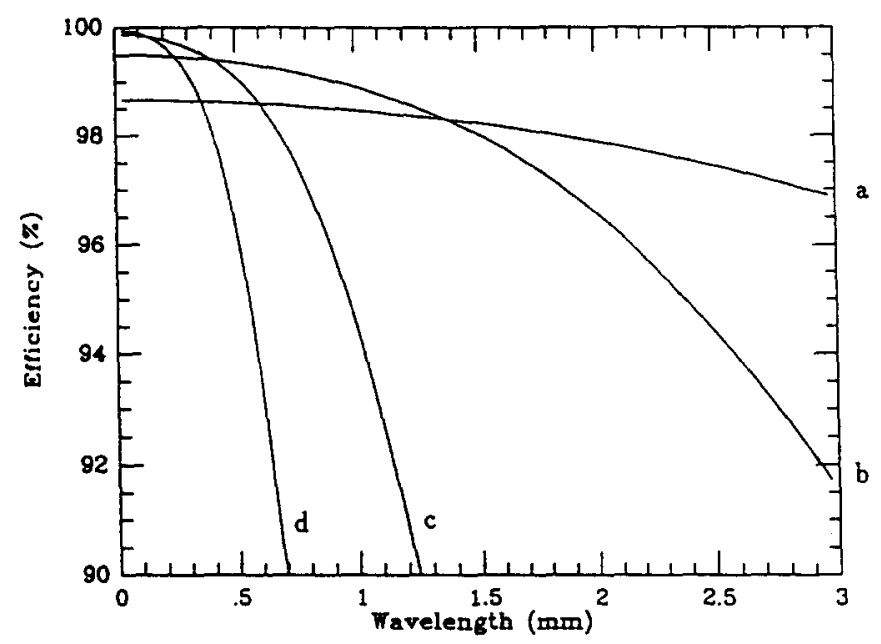

Fig. 5. The coupling efficiency of the lowest-order Gaussian mode as a function of wavelength. The plots correspond to input-beam focal ratios of (a) 3, (b) 5, (c) 10 and (d) 15. A paraboloidal mirror having a focal length of $50 \mathrm{~mm}$ and an angle of incidence of $45^{\circ}$ was used throughout.

least; at short wavelengths, it is possible to pass an almost collimated beam through a mirror without projection loss.

In Fig. 5, we show the coupling efficiency of the lowest-order mode as a function of wavelength for various input-beam focal ratios. A mirror having a focal length of $50 \mathrm{~mm}$ was used throughout. At short wavelengths, the mirror behaves in an almost ideal fashion, but at long wavelengths, the efficiency falls due to phase-error and projection errors. As the wavelength increases, the large-focal-ratio beams lose efficiency more quickly because they move into the near field of the illuminating beam more quickly; the projection losses also increase due to the focal ratio of the output beam becoming smaller-actually, the long-focal-length beams start to violate the condition described in the previous section. In addition, projection effects can be seen at short wavelengths, and it seems that they are worse for beams having large opening angles and large angles of incidence. It is interesting to note that if we take a cut through the curves at a given frequency-that is to say is we plot the coupling efficiency as a function of the focal ratio-the projection losses are least when the waist at the centre of the mirror is smallest, or equivalently when the focal ratios of the input and output beams are equal.

In summary, parabolic mirrors can be used to make Gaussian beam telescopes as long as the mirrors are essentially, but not precisely, in the far-fields of the illuminating beams at the longest wavelength: $f>z c=4 \lambda f b^{2} / \pi$.

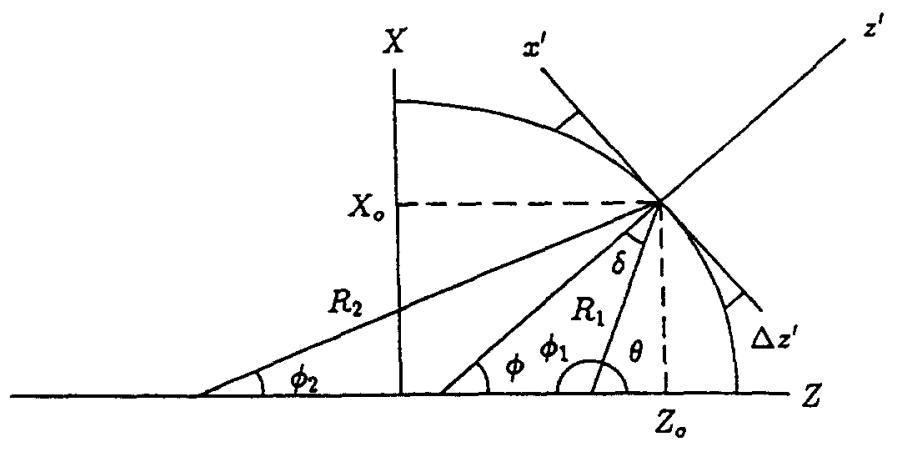

Fig. 6. The geometry of an offset ellipsoidal reflector. 


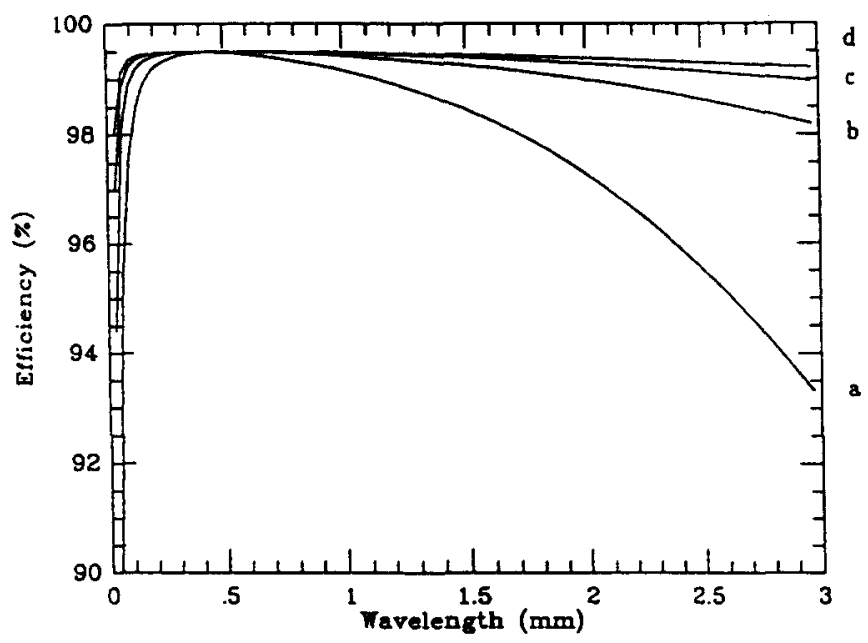

Fig. 7. The coupling efficiency of the lowest-order Gaussian mode as a function of wavelength. The plots correspond to offset ellipsoidal reflectors having focal lengths of (a) $50 \mathrm{~mm}$, (b) $100 \mathrm{~mm}$, (c) $150 \mathrm{~mm}$ and (d) $200 \mathrm{~mm}$. The focal ratio of the illuminating beam is held constant at five, the input waist is at the focus, the angle of incidence is $45^{\circ}$, and the design wavelength is $0.63 \mathrm{~mm}$.

\section{ELLIPSOIDAL MIRRORS}

When manufacturing Gaussian-beam telescopes for long wavelengths, it is desirable to use ellipsoidal mirrors because they have two characteristic radii of curvature. The usual approach is to set one characteristic radius $R l$ equal to the radius of curvature of the input beam, and the other characteristic radius $R 2$ equal to the radius of curvature of the output beam. This optimization can, however, only be done at one particular frequency.

To analyse ellipsoidal mirrors we consider the geometry shown in Fig. 6. The major and minor axes of the parent ellipsoid are given by

$$
a=\frac{(R 1+R 2)}{2} \text { and } b=\sqrt{R 1 R 2} \cos \delta
$$

We now need to determine the angle of the normal to the tangent plane with respect to the axis of rotation. Consideration of the geometry shows that

$$
\tan \phi 1=\frac{\sin (2 \delta)}{\left[\frac{R 1}{R 2}-\cos (2 \delta)\right]},
$$

and also $\phi=\pi-\phi 1-\delta$ and $\theta=\pi-\phi 1$. The vertex of the mirror is at

$$
X_{o}=R 1 \sin \theta \quad \text { and } Z_{o}=R 1 \cos \theta+a e,
$$

where $e$ is the eccentricity. Once again by shifting and rotating the $(X, Z)$ co-ordinate frame, it can be shown that the height of the surface above the tangent plane is given by equation (25) with

$$
\begin{aligned}
& A=\frac{\sin ^{2} \phi}{b^{2}}+\frac{\cos ^{2} \phi}{a^{2}} \\
& B=\frac{2 x_{o} \cos \phi \sin \phi}{\cos \delta}\left(\frac{1}{b^{2}}-\frac{1}{a^{2}}\right)+\frac{2 X_{o} \sin \phi}{b^{2}}+\frac{2 Z_{o} \cos \phi}{a^{2}} \\
& C=\frac{y_{o}^{2}}{b^{2}}+\left(\frac{x_{o}}{\cos \delta}\right)^{2}\left(\frac{\cos ^{2} \phi}{b^{2}}+\frac{\sin ^{2} \phi}{a^{2}}\right)+2\left(\frac{x_{0}}{\cos \delta}\right)\left(\frac{\cos \phi X_{o}}{b^{2}}-\frac{\sin \phi Z_{o}}{a^{2}}\right) .
\end{aligned}
$$




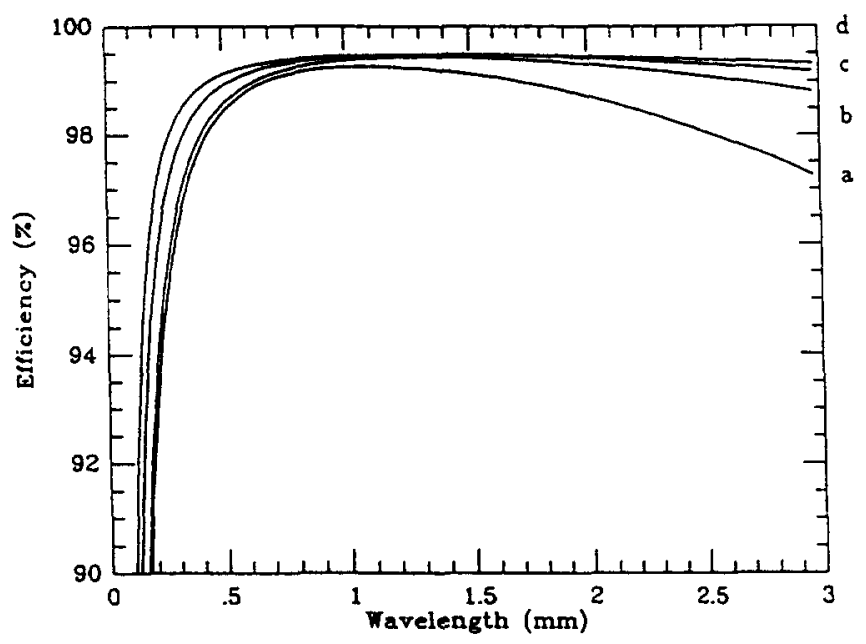

Fig. 8. The coupling efficiency of the lowest-order Gaussian mode as a function of wavelength. The plots correspond to offset ellipsoidal reflectors having focal lengths of (a) $50 \mathrm{~mm}$, (b) $100 \mathrm{~mm}$, (c) $150 \mathrm{~mm}$ and (d) $200 \mathrm{~mm}$. The focal ratio of the illuminating beam is held constant at five, the input waist is at the focus, the angle of incidence is $45^{\circ}$, and the design wavelength is $2.0 \mathrm{~mm}$.

For the purpose of finding the position of the output waist, we define the focal length of the mirror to be $f=R 1 R 2 /(R 1+R 2)$.

In Fig. 7, we show the coupling efficiency of the lowest-order Gaussian mode as a function of wavelength for ellipsoidal mirrors having focal lengths of 50, 100, 150 and $200 \mathrm{~mm}$. Again the focal ratio of the input beam is held constant at $f b=5$. The situation is the same as that of the paraboloidal mirror, but now we introduce an optimum design wavelength of $0.63 \mathrm{~mm}$. The results shown in Fig. 7 are similar to those of the paraboloidal mirror, Fig. 4, but now we find that modest improvements are made at long wavelengths at the expense of dramatic losses in efficiency at short wavelengths.

In Fig. 8 we show a similar set of plots, but in this case the mirrors are designed for a centre wavelength of $2.0 \mathrm{~mm}$. As expected, the short-wavelength fall off occurs at a lower frequency, and the long-wavelength efficiency of the short-focal-length mirror is improved. It is notable that the

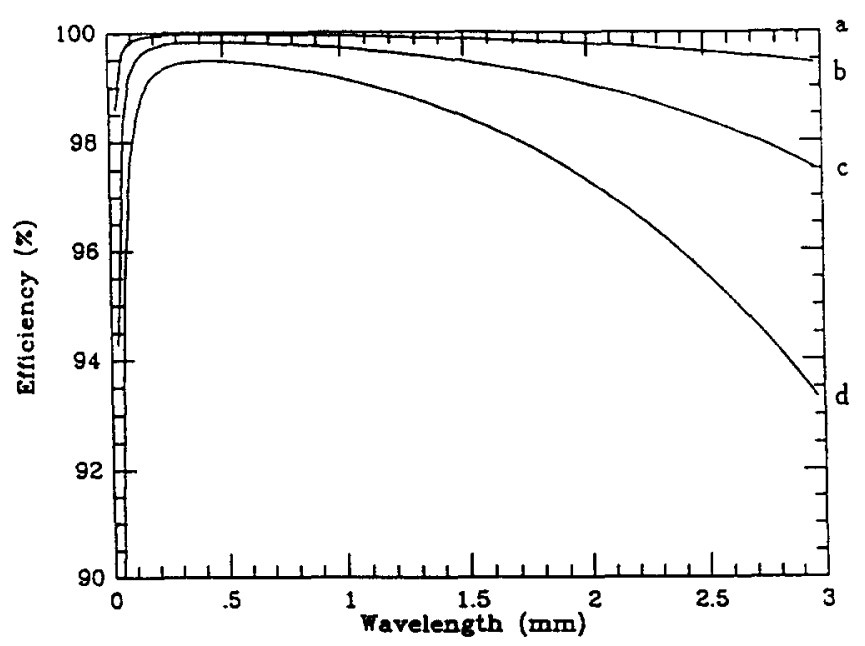

Fig. 9. The coupling efficiency of the lowest-order Gaussian mode as a function of wavelength. The plots correspond to angles of incidence of (a) $0^{\circ}$, (b) $15^{\circ}$, (c) $30^{\circ}$ and (d) $45^{\circ}$. An offset ellipsoidal reflector having a focal length of $50 \mathrm{~mm}$ was used. The design wavelength is $0.63 \mathrm{~mm}$ and the focal ratio of the illuminating beam is five. 
efficiency only peaks at the design wavelength when the focal length of the mirror is reasonably large. This occurs because projection effects influence the overall behaviour. As with the parabolic mirrors, our assumptions tend to exaggerate projection losses.

To complete the section, we show in Fig. 9 the effect of tilting a mirror. For this example, we have used an ellipsoidal mirror having a focal length of $50 \mathrm{~mm}$ and designed for a centre wavelength of $0.63 \mathrm{~mm}$. Clearly, for any mirror there is a maximum efficiency, which is determined by the form of the beam and the angle of incidence. As the mirror is turned upright, the bandwidth of the system increases. Indeed, it is possible to show analytically that, at any wavelength, the losses should change as $\tan ^{2} \delta$, and this is born out by our simulations.

\section{MEASURED SURFACES}

As the shape of a mirror is characterized by the height of its surface above a tangent plane, it is straightforward to make measurements on an actual surface and from these get an indication of performance. In Fig. 10, we show plots of the calculated performance of an off-axis ellipsoidal mirror of focal length $350 \mathrm{~mm}$. The mirror surface was measured over a grid of points separated by $10 \mathrm{~mm}$, and a bicubic-spline interpolation routine was used in the mirror software to generate an approximation to the true surface. We know that this mirror has large-scale surface errors of $20 \mu \mathrm{m}$. The low-frequency fall off in efficiency is not due to the surface of the mirror but is due to truncation at the edges-our mirror was actually designed to accommodate an image rather than just the lowest-order Gaussian mode. This explanation is verified by comparing the efficiency of the measured surface with that expected from an ideal truncated surface. The important observation is that, at high frequencies, the performance of the real mirror deteriorates at a wavelength consistent with the known surface errors.

\section{CONCLUDING REMARKS}

We have presented an approximate technique, based on the principles of Gaussian-beam optics, for calculating the performance of shaped off-axis mirrors. In principle, the technique is capable of accounting for three types of error: amplitude errors due to projection effects associated with the inclination of the surface; phase errors associated with the spherical phase fronts of the

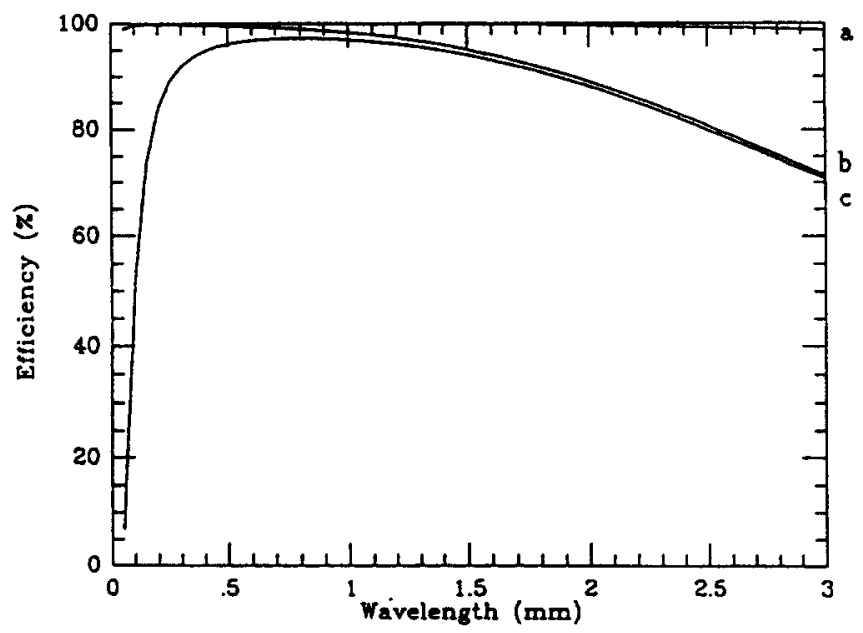

Fig. 10. The coupling efficiency of the lowest-order Gaussian mode as a function of wavelength. Curve (a) shows the performance of an ideal ellipsoidal surface having a focal length of $350 \mathrm{~mm}$; curve (b) shows the performance of an ideal but truncated surface of the same focal length; and curve (c) shows the performance of a measured surface, which should have behaved in the same way as curve (b). The reduction in efficiency at short wavelengths is due to large-scale surface errors of $20 \mu \mathrm{m}$. 
incoming and outgoing beams not being matched; and phase errors due to the mirror occupying a region in space in which the beams are diffracting.

The approximations underlying the technique are not severe, and we believe that we have a good first-order description of the coupling process. It must be said, however, that at the present time we do not fully understand the capabilities and idiosyncracies of the method. For example, phase errors in general will lead to skewing and spreading of the reflected beam, and it is not clear how well these fields can be described by a small number of Gaussian modes. That is to say, it is not clear how well the sum of the modes reproduces the actual aberrated image. Our next task is to compare more thoroughly the predictions of our technique with those of classical theory.

\section{REFERENCES}

1. P. F. Goldsmith, Infrared and Millimeter Waves, Vol. 6, p. 277. Academic Press, New York (1982).

2. D. H. Martin and J. Lesurf, Infrared Phys. 18, 405 (1978).

3. A. W. Rudge and N. A. Adatia, Proc. IEEE 66, 1592 (1978).

4. H. Matsuura and K. Hongo, IEEE Trans. Antennas Propagat. AP-35, 101 (1987).

5. T. S. Chu, IEEE Trans. Antennas Propagat. AP-31, 6 [4 (1983).

6. S. Withington, J. A. Murphy, A. Egan and R. E. Hills, Infrared Millimeter Waves 13, 1515 (1992).

7. K. G. Isaak and S. Withington, Proc. 4th Int. Symp. Space Terahert= Technology, UCLA, Los Angeles. p. 199 (1993).

8. J. A. Murphy and $S$. Withington, In preparation.

9. J. Touvinen, IEEE Trans. Antennas Propagat. 40, 391 (1992). 